\title{
SALMONELLA OSTEOMYELITIS OF THE LUMBAR SPINE: AN UNUSUAL PRESENTATION IN AN IMMUNOCOMPETENT MALE
}

\author{
AROOP MOHANTY ${ }^{1 *}$, NAVEEN PANDITA ${ }^{2}$, PANKAJ KANDWAL ${ }^{2}$, BALRAM JI OMAR ${ }^{1}$, PRATIMA GUPTA ${ }^{1}$, \\ PRIYANKA GUPTA ${ }^{1}$
}

\begin{abstract}
${ }^{1}$ Department of Microbiology, All India Institute of Medical Sciences, Rishikesh, Uttarakhand, India. ${ }^{2}$ Department of Orthopedics, All India Institute of Medical Sciences, Rishikesh, Uttarakhand, India. Email: aroopmohanty7785@yahoo.com
\end{abstract}

Received: 19 Septemper 2017, Revised and Accepted: 09 October 2017

ABSTRACT

Prevalence of Salmonella vertebral osteomyelitis has increased in recent years due to greater number of spinal surgical procedures, ageing population, and intravenous drug abuse. It is a rare complication of Salmonella infection. We report a case of a 17-year-old male who presented with low back pain for past 1 month. Physical examination revealed spinal tenderness over L2-L5 spine with sensory and motor deficit. Magnetic resonance imaging of dorsolumbar spine showed spondylodiscitis at L2-L5 and epidural collection at L4-L5 level. The patient did not respond to conservative treatment and trial of antitubercular drugs. He underwent open discal biopsy and decompression laminotomy. Intraoperatively, he was found to have epidural abscess and discitis at L3-L4 level. Tissue and wound culture grew Salmonella typhi, and with antibiotic susceptibility guidance, he was treated with intravenous cefuroxime for 4 weeks. On his latest follow-up, there was complete recovery and fusion at diseased vertebrae level.

Keywords: Spondylodiscitis, Salmonella typhi, Back pain.

(c) 2018 The Authors. Published by Innovare Academic Sciences Pvt Ltd. This is an open access article under the CC BY license (http://creativecommons. org/licenses/by/4. 0/) DOI: http://dx.doi.org/10.22159/ajpcr.2018.v11i1.22530

\section{INTRODUCTION}

Spondylodiscitis is a diagnosis rarely made in the community and emergency medicine. Although neck pain and back pain are common presentations, spondylodiscitis is usually not among the forefront of differential diagnoses. Its incidence has been estimated at 2.4 cases per 100,000 population, which increases with age (from 0.3 per 100,000 in the $<20$ years age group to 6.5 per 100,000 in the over 70 years group) [1]. S. typhi, apart from presenting in its unusual classical domain as an enteric pathogen, may present as localized infection at almost any site in the body. It is responsible for variety of human illnesses ranging from gastroenteritis, typhoid fever, and bacteremia to the asymptomatic carrier state [2]. Infection of a spinal segment with paravertebral abscess due to Salmonella has been reported to be extremely rare and that too in patients with predisposing factors such as sickle cell anemia, corticosteroid therapy, and malignancies $[3,4]$. Since the incidence of tubercular infection of the spine is very high in the Indian subcontinent, it leads to a high index of suspicion and institution of empirical treatment for the same. Here, we present a case of vertebral osteomyelitis whereby a presumptive diagnosis of tubercular infection was made, but the microbiology investigation of the pus culture from the intervertebral disc yielded S. typhi.

\section{CASE REPORT}

A 17-year-old male patient presented to orthopedics OPD, with complaints of backache located in lumbar region for past 1 month, it was nonradiating in nature with reduced sensation bilaterally in lower limbs. Pain was relieved on lying in prone position, whereas it got aggravated in supine and standing position. On local and general physical examination, the patient was afebrile with excessive paraspinal muscle spasm and lumbar lordosis. On palpation, there was severe tenderness over L2-L5 region with restriction of movements. Neurological examination revealed sensory deficit below L4 level bilaterally and motor Grade 4 power bilaterally in L4 and L5. There was neither bowel bladder involvement nor saddle anesthesia. The total leucocyte count was mildly elevated at $12.54 \times 109 / \mathrm{L}$, and ESR and CRP were elevated at $92.5 \mathrm{~mm} / \mathrm{h}$ and $14 \mathrm{mg} / \mathrm{L}$, respectively. Screening for HIV, Hepatitis B, and Hepatitis C markers was negative. Plain X-ray of the thoracic and lumbar spine revealed paradiscal erosions at the level of L3-L4 with the reduction of disc space (Fig. 1). Magnetic resonance imaging (MRI) thoracic and lumbar spine showed spondylodiscitis at L2, L3, L4, and L5 vertebrae and their adjacent disc spaces. There were associated paravertebral and epidural components with compression of the spinal cord (Fig. 2).

On the basis of patient's history and radiological findings, a provisional diagnosis of Pott's spine L3-L4 was made and antitubercular drugs (ATT) started. On the $5^{\text {th }}$ day of admission, the patient had 2-3 episodes of loose stools with blood which subsided on its own. Keeping in mind an abdominal pathology coexisting with the spinal condition, a detailed history was taken. On repeat history taking it was revealed that the patient had fever and abdominal pain 3 months before developing back pain. At that time, he was prescribed antipyretics and analgesics which had provided him with partial relief, but he had subsequently lost appetite and weight. Routine stool examination, typhi dot and blood culture were sent to the department of microbiology. No ova or cyst was seen on stool examination, but surprisingly both blood culture (three different sites) and typhi dot came positive for $S$. typhi in the $2^{\text {nd }}$ week of illness. On the $14^{\text {th }}$ day of admission, the patient underwent open discal biopsy and decompressive laminotomy at (L4-L5) region. Intraoperatively, he was found to have discitis involving L3-L4 and L4-L5 disc space with epidural abscess. Pus aspirated from the disc space and paravertebral area was sent for the presence of AFB/TB culture and routine culture and sensitivity. The pus sample was negative for AFB, but the culture plate grew $S$. typhi sensitive to aminoglycosides, cephalosporins (including ceftriaxone and cefuroxime), and $\beta$-lactam drugs. Mycobacterial culture using Gene X-pert also could not detect MTB. ATT was stopped immediately and the patient was started on injection cefuroxime for 3 weeks on inpatient basis which was continued as oral for a total of 6 weeks. The patient responded to treatment and showed rapid symptomatic and neurological improvement over the course of 6 weeks of follow up. Post-operative inflammatory markers like ESR and CRP came down to $10 \mathrm{~mm} / \mathrm{h}$ and $5.6 \mathrm{mg} / \mathrm{L}$. Follow-up after 6 months showed that the patient was asymptomatic with no 


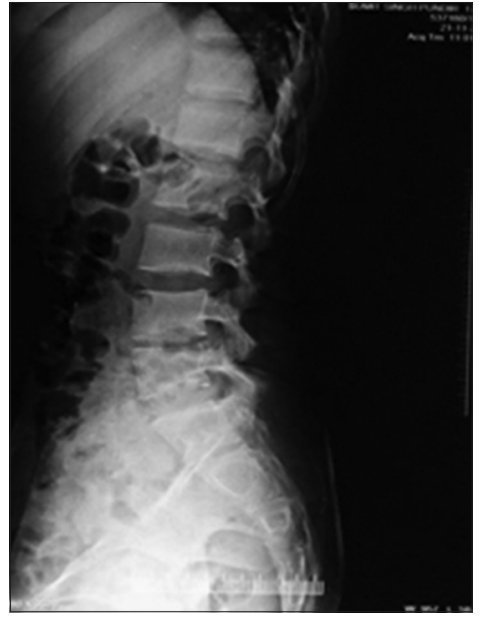

Fig. 1: Pre-operative X-ray L/S spine, lateral view showing reduction of disc space between L4-L5 with irregularity of end plates

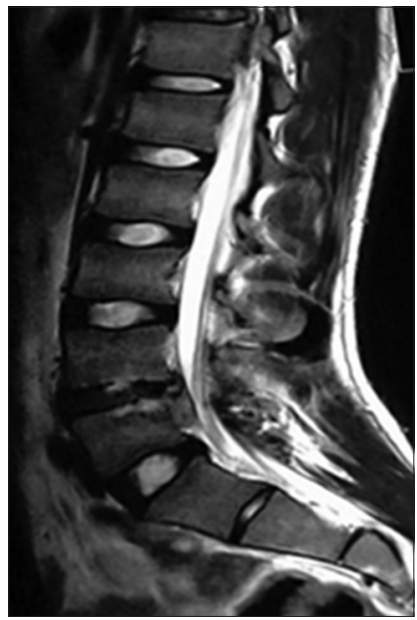

Fig. 2: Pre-operative T2-weighted magnetic resonance imaging of patient showing spondylodiscitis at the level of lumbar spine worse at L4-L5 level with destruction of intervertebral disc and epidural collection and cord compression and edema

neurological deficit. Repeat X-ray was taken which revealed fusion at diseased vertebrae level (Fig. 3).

\section{DISCUSSION}

Salmonella osteomyelitis is uncommon and typhoid osteomyelitis of the spine is even more uncommon [4]. Clinically, Salmonella osteomyelitis is similar to pyogenic vertebral osteomyelitis due to other causes, for example, the bimodal age distribution, male preponderance, predominantly lumbar involvement, and monomicrobial nature of infection [5]. Salmonella infection is a rare cause of osteomyelitis in patients with no sickle cell disease, accounting approximately for $0.5 \%$ of all cases [6]. Our case is a rare presentation as the patient did not have any predisposing factors.

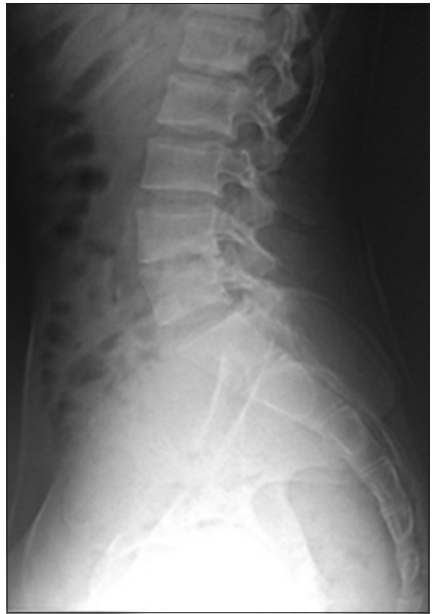

Fig. 3: Post-operative X-ray L/S spine, lateral view showing fusion at diseased vertebrae levelc

Santos and Sapicro reviewed 44 cases of vertebral osteomyelitis and concluded that fever and back pain were the main presenting symptoms [7]. Therefore, febrile patients presenting with back pain should be fully investigated by imaging studies to rule out vertebral osteomyelitis. However, in this case, the patient was afebrile. Thus, one should suspect vertebral osteomyelitis in a patient with back pain, and Salmonella infection should be considered if there has been a recent gastrointestinal episode too. Definitive diagnosis of Salmonella vertebral osteomyelitis is done by isolation of the bacteria from bone specimen obtained by biopsy/aspirate of adjacent fluid collection/ positive blood culture [6]

Tuberculosis infection in India is very common. Hence, a lot of clinicians start empirical ATT on the basis of radiological features; however, this may not relieve the symptoms caused by Salmonella osteomyelitis. Thus, antibiotic therapy is the mainstay of treatment in Salmonella vertebral osteomyelitis, and the choice of drug should be guided by antibiotic susceptibility tests for at least 6 weeks.

\section{REFERENCES}

1. Grammatico L, Baron S, Rusch E, Lepage B, Surer N, Desenclos JC, et al. Epidemiology of vertebral osteomyelitis (VO) in France: Analysis of hospital-discharge data 2002-2003. Epidemiol Infect 2008;136:653-60.

2. Rubin RH, Weinstein L. Salmonellosis: Microbiologic, Pathologic and Clinical Features. New York, NY, USA: Stratton Intercontinental Medical Book Corporation; 1977

3. Abdullah SH, Ata OA, Al-Adwan N. Thoracic spinal epidural abscess caused by Salmonella typhi: Case report. Neurol Med Chir 2008;48 Suppl 3:140-2.

4. Khan FY, El-Hiday AH, Kamel HA. Typhoid osteomyelitis of the lumbar spine. Hong Kong Med J 2006;12:391-3.

5. Carvell JE, Maclarnon JC. Chronic osteomyelitis of the thoracic spine due to Salmonella typhi: A case report. Spine (Phila Pa 1976)1981;6:527-30

6. Gaujoux-Viala C, Zeller V, Leclerc P, Chicheportiche V, Mamoudy P, Desplaces $\mathrm{N}$, et al. Osteomyelitis in adults: An under recognized clinical entity in immunocompetent hosts. A report of six cases. Joint Bone Spine 2011;78:75-9.

7. Santos EM, Sapico FL. Vertebral osteomyelitis due to salmonellae: Report of two cases and review. Clin Infect Dis 1998;27:287-95. 